\title{
Memory effect in Yang-Mills theory
}

\author{
Niko Jokela, ${ }^{1,2, *}$ K. Kajantie, ${ }^{2, \dagger}$ and Miika Sarkkinen ${ }^{1, \$}$ \\ ${ }^{1}$ Department of Physics, University of Helsinki, FI-00014 Finland \\ ${ }^{2}$ Helsinki Institute of Physics, University of Helsinki, FI-00014 Finland
}

(Received 2 April 2019; published 6 June 2019)

\begin{abstract}
We study the empirical realization of the memory effect in Yang-Mills theory, especially in view of the classical vs quantum nature of the theory. Gauge invariant analysis of memory in classical U(1) electrodynamics and its observation by total change of transverse momentum of a charge is reviewed. Gauge fixing leads to a determination of a gauge transformation at infinity. An example of Yang-Mills memory then is obtained by reinterpreting known results on interactions of a quark and a large high energy nucleus in the theory of color glass condensate. The memory signal is again a kick in transverse momentum, but it is only obtained in quantum theory after fixing the gauge, after summing over an ensemble of classical processes.
\end{abstract}

DOI: 10.1103/PhysRevD.99.116003

\section{INTRODUCTION}

The memory effect in gravitational radiation $[1,2]$ is the total change in the positions (or other properties) of a system of detectors left by a burst of gravitational radiation. Conceptually, the detectors lie at null infinity and that is where massless gravitons end up. The effect can also be formulated for other massless (or nearly massless) particles like photons [3,4] or neutrinos [5]. Massless quanta exist also in Yang-Mills (YM) theories, and color memory has been studied in [6] in a classical perturbative approximation, for which the discussion of electrodynamics [7] can directly be extended.

Yang-Mills fields form an integral part of the standard model (SM), SU(3) invariance in the strong quark-gluon sector and $\mathrm{SU}(2) \times \mathrm{U}(1)$ invariance in the electroweak sector. However, in both cases the theory is in a phase which, apart from the photon, does not contain massless particles to be sent to null infinity. In fact, this is the very reason for their being the SM. The $\mathrm{SU}(3)$ sector is in the confinement phase; massless gluons do not propagate but develop a gap, become massive glueballs and do not go to null infinity. The $\mathrm{SU}(2) \times \mathrm{U}(1)$ sector is in a Higgs phase, the candidate gluons develop mass via the Higgs mechanism and, after mixing with $\mathrm{U}(1)$, become three massive

\footnotetext{
"niko.jokela@helsinki.fi

†keijo.kajantie@helsinki.fi

¥miika.sarkkinen@helsinki.fi
}

Published by the American Physical Society under the terms of the Creative Commons Attribution 4.0 International license. Further distribution of this work must maintain attribution to the author(s) and the published article's title, journal citation, and DOI. Funded by SCOAP. vector bosons and the single massless photon. For a lucid comparative exposition of the SM confinement and Higgs phases, see [8].

There is one phenomenological context in which classical Yang-Mills fields are studied with some justification: the wave function of a heavy nucleus when probed with a large scale probe like an electron in deep inelastic scattering [9]. A large nucleus probed with large $Q^{2}$ involves large occupation numbers and hence classical fields. The realization of the ideas in [6,7] in this context has already been studied in [10]. We wish in this paper to give a simple discussion of YM memory in the spirit of $[3,4]$, emphasizing the fact that any experimental measurement of the suggested YM effect is inherently quantum mechanical. Here the enormous nonlinear complexity of classical theory is replaced by a relative simplicity of quantum mechanical expectation values. As a background we first discuss the memory in electrodynamics and explain why we feel that the "new symmetries of QED" [7] are basically U(1) invariance of classical ED, when applied in a fixed gauge at null infinity.

Actually the place where $\mathrm{SU}(3) \mathrm{YM}$ memory effect empirically appears is very easy to locate and well known in the field, just the nomenclature has to be changed. For example, Fig. 12 of [9] shows how a passage of a large energy nucleus creates from vacuum a transverse matrix color field $A^{i}, i=2,3$, also gauge equivalent to vacuum, $F_{i j}=0$. The nucleus is the analogue of a burst of YM radiation, the color field $A^{i}$ is the analogue of its memory or of the "large" gauge transformation in [6]. More precisely, $A^{i}$ is the (square root of) the quantum expectation value of the square of the YM field, summed over colors. Quantum physics cannot be avoided.

A basic memory signal will be transverse momentum kick of a test object. In $\mathrm{U}(1)$ one is hereby done, no 
problem in measuring the kick. In YM one further has to see how this kick is measured. These measurements are rather indirect, as thoroughly discussed in [10]. Another possibility would be to use the gluon radiation from the acceleration of the test quark, as recently computed in [11]. The use of YM memory as an analogue of gravitational wave memory is thus very limited.

The electroweak sector of the SM can also be forced out of the Higgs phase by similar means, by large occupation numbers and associated classical fields in the very early Universe. The boson equilibrium occupation number at small $k$ is $n=1 /\left(e^{k / T}-1\right) \sim T / k \sim 1 / g^{2} \gg 1$ since the dominant infrared scale is the coupling of the $3 \mathrm{D}$ magnetic sector of the theory, $k \sim g^{2} T$. Important physical phenomena like baryon number violation rates in the SM can be numerically studied in this setting; see for example $[12,13]$. A study of YM memory also for these fields should be possible, also in an expanding universe $[14,15]$.

In discussions of memory effect one usually thinks about radiation propagating over huge distances, millions of light years. In the heavy ion Yang-Mills case one clearly must be content with much smaller distances. This is related to the coupling not being asymptotically small at the relevant scale of about $1 \mathrm{GeV}$. Phenomenologically the coupling constant $g(\mu=1 \mathrm{GeV}) \approx 2$ is actually "large" in the usual $\overline{\mathrm{MS}}$ renormalization scheme in the sense that the distance scale generated by renormalization is "small". For $N_{c}=N_{f}=3$,

$$
\frac{1}{\Lambda_{\mathrm{QCD}}}=\frac{1}{\mu} \exp \left[\frac{8 \pi^{2}}{9 g^{2}(\mu)}\right]\left(\frac{9 g^{2}}{16 \pi^{2}}\right)^{\frac{32}{81}} \approx 1 \mathrm{fm} .
$$

Theoretically, of course one can apply much smaller values of $g^{2}$, and there is underway an intense numerical effort for studying classical YM equations in the weak coupling region (see, for example, [16,17]). In the SM, the corresponding $\mathrm{SU}(2)$ coupling constant $g(\mu=100 \mathrm{GeV}) \approx 2 / 3$ is actually small in the sense that the corresponding distance scale is macroscopic. Including just the 2-loop running of the $\mathrm{SU}(2)$ coupling [18], the renormalization group integration constant is

$$
\frac{1}{\Lambda_{2}}=\frac{1}{\mu} \exp \left[\frac{48 \pi^{2}}{19 g^{2}(\mu)}\right]\left(\frac{19 g^{2}}{96 \pi^{2}}\right)^{\frac{483}{361}} .
$$

Putting here $g(\mu=100 \mathrm{GeV})=\frac{2}{3}$ gives an $\mathrm{SU}(2)$ distance scale of about $1 / \Lambda_{2}=4000 \mathrm{~km}$, a macroscopic distance. However, the standard model is in the Higgs phase so that this is not a proper confinement radius within which the fields would be massless.

In the following we shall limit ourselves to a summary of the memory in U(1) ED (Sec. II) and a discussion of memory in SU(3) heavy ion collisions (Secs. III and IV). Note on angular coordinates at large distances: for ED we use two angular coordinates $\theta_{A}=h_{A B} \theta^{B}$ on $S^{2}$, while for the heavy ion case we use two Cartesian coordinates $x_{i}=x^{i}, i=2,3$ transverse to the beam direction $x^{1}$. We use the mostly plus metric; its advantage is that one can write $x_{i}=x^{i}$ without a sign change.

\section{MEMORY IN ELECTRODYNAMICS}

For ED everything follows from Maxwell's equations, evaluated at $\mathcal{I}^{+}$, future null infinity. The essence of the phenomenon can be summarized as follows, in the spirit of [3].

We use the coordinates $t, r, \theta^{A}$ and the metric,

$g_{\mu \nu}=\left(\begin{array}{ccc}-1 & 0 & 0 \\ 0 & 1 & 0 \\ 0 & 0 & r^{2} h_{A B}\end{array}\right), \quad g^{\mu \nu}=\left(\begin{array}{ccc}-1 & 0 & 0 \\ 0 & 1 & 0 \\ 0 & 0 & h^{A B} / r^{2}\end{array}\right)$,

where $h_{A B}$ is the metric on the celestial sphere $\mathrm{S}^{2}$, with, for example, $\theta^{A}=(\theta, \phi)$ in standard spherical coordinates or $\theta^{A}=\left(z=e^{i \phi} / \tan (\theta / 2), \bar{z}\right)$ in stereographic coordinates. Maxwell's equations are

$$
\begin{gathered}
\nabla_{\mu} F^{\mu \nu}=\frac{1}{\sqrt{-g}} \partial_{\mu}\left(\sqrt{-g} F^{\mu \nu}\right)=J^{\nu} \\
\nabla_{\alpha} F_{\beta \gamma}+\nabla_{\beta} F_{\gamma \alpha}+\nabla_{\gamma} F_{\alpha \beta}=\partial_{\alpha} F_{\beta \gamma}+\partial_{\beta} F_{\gamma \alpha}+\partial_{\gamma} F_{\alpha \beta}=0 .
\end{gathered}
$$

Splitting vectors in their radial and celestial sphere $\mathrm{S}^{2}$ components, $E^{a}=\left(E^{r}, E^{A}\right), E^{A}=h^{A B} E_{B} / r^{2}$, the inhomogeneous (2.2) and homogeneous equations (2.3) are

$$
\begin{aligned}
J^{t} & =\frac{1}{r^{2}} \partial_{r}\left(r^{2} E_{r}\right)+D_{A} E^{A}, \\
J^{r} & =-\partial_{t} E_{r}+\epsilon^{A B} D_{A} B_{B}, \\
J_{A} & =-\partial_{t} E_{A}+\epsilon_{A}^{B}\left(\partial_{B} B^{r}-\partial_{r} B_{B}\right) . \\
0 & =\frac{1}{r^{2}} \partial_{r}\left(r^{2} B_{r}\right)+D_{A} B^{A}, \\
0 & =\partial_{t} B^{r}+\epsilon^{A B} D_{B} E_{A}, \\
0 & =\partial_{t} B_{A}-\epsilon_{A}^{B}\left(\partial_{B} E_{r}-\partial_{r} E_{B}\right),
\end{aligned}
$$

where the $2 \mathrm{D}$ epsilon tensor is $\left(h=\operatorname{det} h_{A B}\right)$,

$$
\begin{aligned}
\epsilon_{a b} & =\sqrt{|h|}\left(\begin{array}{cc}
0 & 1 \\
-1 & 0
\end{array}\right) \equiv \sqrt{|h|} \eta_{a b} . \\
\epsilon^{a b} & =\frac{1}{h} \epsilon_{a b}=\frac{\operatorname{sign} h}{\sqrt{|h|}}\left(\begin{array}{cc}
0 & 1 \\
-1 & 0
\end{array}\right)=\frac{\operatorname{sign} h}{\sqrt{|h|}} \eta_{a b} .
\end{aligned}
$$

To analyze the memory we need a radial current at null infinity, i.e., 


$$
J^{t}=\rho=\frac{L\left(u, \theta^{A}\right)}{r^{2}}=J^{r}, \quad u=t-r
$$

The $r$ dependence is constrained by the total luminosity $\sim L\left(u, \theta^{A}\right)$ being finite. The transverse current can be negligible, i.e., $J_{A} \sim 1 / r^{3}$. Note that this current pattern rigorously speaking implies that we should have massless charged particles, since only they get to null infinity. This is a practical issue, though. For observations null infinity is at a finite distance.

We now expect at null infinity a typical radiation pattern with transverse and orthogonal electric and magnetic fields. To analyze the magnitudes it is enough to focus on the Gauss' law, the first equation in (2.4). Canceling a common factor $1 / r^{2}$, replacing $\partial_{r} \rightarrow-\partial_{u}$ (at $\mathcal{I}^{+}$fields are functions of $t-r$ ) and letting $E_{r}$ denote the leading $r$ behavior, $E_{r}^{(2)}=r^{2} E_{r} \rightarrow E_{r}$ the equation is

$$
-\partial_{u} E_{r}+D_{A} E^{A}=L .
$$

Integrating this over $u_{i}<u<u_{f}$ gives

$$
E_{r}\left(u_{i}\right)-E_{r}\left(u_{f}\right)+D_{A} \int d u E^{A}=\int d u L\left(u, \theta^{A}\right) \equiv F\left(\theta^{A}\right),
$$

which we write in the form

$$
D_{A} M^{A}=\Delta E_{r}+F\left(\theta^{A}\right),
$$

defining the fundamental quantity, the memory vector,

$$
M_{A}\left(\theta^{A}\right)=\int_{u_{i}}^{u_{f}} d u E_{A}
$$

The memory vector represents the cumulative effect on the celestial sphere of a pulse of radiation sent to $\mathcal{I}^{+}$. Its significance is based on the fact that it is measurable. Integrating the Lorentz force equation

$$
\frac{d p^{\mu}}{d \tau}=q F^{\mu \nu} u_{\nu}, \quad p^{\mu}=m u^{\mu},
$$

for $\mu=B$ and for small velocities over time (effectively the same as $u$ ) the change of the transverse momentum is

$$
\int d t \frac{d p_{B}}{d t}=\Delta p_{B}=q \int d t E_{B}(t)=q M_{B}\left(\theta^{A}\right) .
$$

The pulse changes the momentum of a test particle by an amount given by the memory vector. This transverse kick is the simplest version of the electromagnetic memory effect. It has two parts, an ordinary kick due to the change of the radial component of the electric field and a null kick due to flux of charge to null infinity, see Fig. 1.

Integrating (2.10) over the celestial sphere, defining $\langle O\rangle=\int d \Omega O$, one has

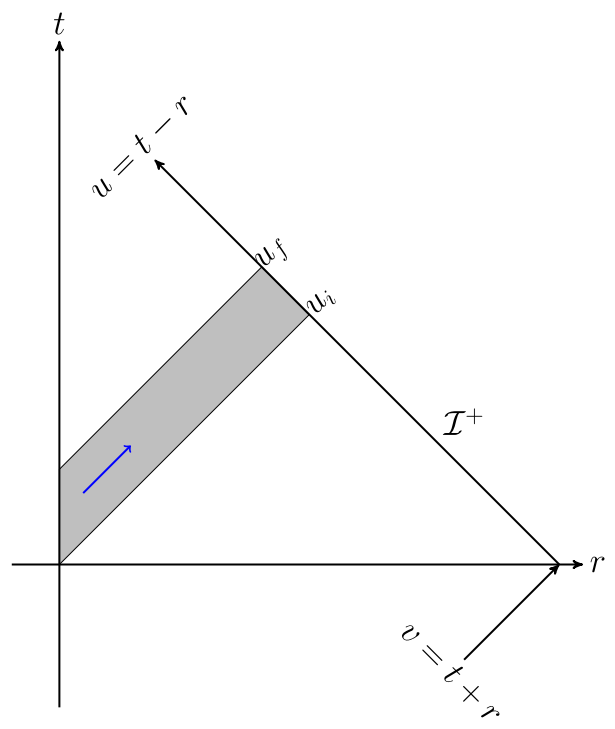

FIG. 1. Memory effect in electrodynamics. A radiator at $r=0$ sends a pulse of radiation to null infinity $\mathcal{I}^{+}$during the time interval $u_{i}<u<u_{f}$. The time integrated pulse of a transverse electric field gives a total momentum kick in (2.13) to a test charge at null infinity.

$$
\begin{aligned}
\left\langle D_{A} M^{A}\right\rangle & =0=\left\langle E_{r}\left(u_{f}\right)\right\rangle-\left\langle E_{r}\left(u_{i}\right)\right\rangle+\langle F\rangle \\
& =Q_{f}-Q_{i}+\langle F\rangle .
\end{aligned}
$$

Here the first step is the fact that an integral of the divergence of a vector field over $S^{2}$ vanishes. Further one uses Gauss law, the integral of $r^{2} E_{r}$ gives the charge inside the sphere. The equation thus expresses the fact that the $L(u)$ term has carried through the sphere the amount $Q_{i}-Q_{f}$ of charge.

Given the charge density $L\left(u, \theta^{A}\right)$ at $\mathcal{I}^{+}$and the change in the radial electric field (here a standard example is a charged particle initially at rest and then moving with constant velocity) one can compute the memory vector, as concretely discussed in [3].

Note that the above discussion is entirely covariant and in terms of fields, vector potentials with some gauge choice have not been used. Consider, however, what happens if one uses the coordinates $u, r, \theta^{A}$ and chooses the temporal gauge $A_{u}=0$ (equivalently, one could choose $A_{r}=0$ and further $A_{u}=0$ at a fixed value of $r, r=\infty$, at $\mathcal{I}^{+}$) [4]. Then $E_{A}=F_{u A}=\partial_{u} A_{A}-\partial_{A} A_{u}=\partial_{u} A_{A}$ and the memory vector and the associated kick are, from (2.13),

$$
\begin{aligned}
\Delta p_{B} & =q M_{B}=q \int d u E_{B}=q \int d u \partial_{u} A_{B} \\
& =q\left(A_{B}\left(u_{f}, \theta^{A}\right)-A_{B}\left(u_{i}, \theta^{A}\right)\right) .
\end{aligned}
$$

We have thus used a physical measurement to determine a gauge choice dependent quantity. Nothing has happened to the symmetry properties of electrodynamics; it is still $\mathrm{U}(1)$ gauge invariant. Interpreted as asymptotic symmetries at null infinity these can be described as new symmetries [7], 
but physically there is nothing beyond $\mathrm{U}(1)$ gauge invariance.

In the literature there are no suggestions of how to realize the ED kick memory in an experimental setup. However, even at a theoretical level it serves to elucidate some aspects of gravitational radiation memory that have remained unclear until recent years. In particular, it serves to underline the fact that there is a distinction between memory due to sources that do not get to null infinity and sources that do, or between ordinary and null memories respectively. Therefore, it may be useful to outline the relation of the above to gravitational radiation and its memory effect. What is the "gauge invariance", how is the gauge "fixed", and what are the "gauge transformations" the parameters of which are determined by measuring the memory effect?

Gauge invariance obviously is the diffeomorphism invariance of general relativity, and gauge fixing is finding the metric containing gravitational radiation, exactly as the Schwarzschild metric contains a black hole. Using the coordinates $u, r, z, \bar{z}$, where $z, \bar{z}$ are the standard stereographic coordinates on the celestial sphere $S^{2}$, this is the Bondi metric [19],

$$
\begin{aligned}
& g_{\mu \nu}=\left(\begin{array}{cccc}
-1+\frac{2 G m(u)}{r} & -1 & u_{z}(z, \bar{z}, u) & u_{\bar{z}}(z, \bar{z}, u) \\
-1 & 0 & 0 & 0 \\
u_{z}(z, \bar{z}, u) & 0 & r c(z, \bar{z}, u) & r^{2} \gamma \\
u_{\bar{z}}(z, \bar{z}, u) & 0 & r^{2} \gamma & r \bar{c}(z, \bar{z}, u)
\end{array}\right) \text {, } \\
& \gamma=\frac{2}{(1+z \bar{z})^{2}}, \quad u_{z}=\frac{1}{2 \gamma} \partial_{\bar{z}} c,
\end{aligned}
$$

$u_{\bar{z}}$ is defined similarly in terms of $\bar{c}$. This metric is defined near null infinity, $u=$ constant, $r \rightarrow \infty$ and contains subtle large $r$ corrections to the flat metric, a Schwarzschildmetric-like but time dependent mass term (which leads to the Vaidya model) and a $1 / r$ correction to the $S^{2}$ metric, specified by the functions $c(z, \bar{z}, u), \bar{c}(z, \bar{z}, u)$. Time dependence of the mass represents flux of gravitational radiation to null infinity. The "gauge transformations" are now those coordinate transformations which leave this Bondi form invariant, zeroes in the metric remain zeroes and $1 / r$ terms get corrections of the same order (so that $m$, $c, \bar{c}$ transform). Sending a pulse of gravitational radiation or total energy $m\left(u_{i}\right)-m\left(u_{f}\right)$ to null infinity will change the functions $c, \bar{c}$ by a calculable amount. This corresponds to a change in the geodesic deviation of two objects at null infinity. This is measurable by the gravitational memory effect so that one thus has measured the parameters of a gauge transformation.

What thus makes the gravitational memory effect physically significant is its direct connection to basic symmetries of GR. In view of this it is notable that the experimental prospects of measuring gravitational memory are quite promising. Even though a direct detection of memory from a single gravitational wave event by LIGO is unlikely (the memory signal $\sim 10^{-1}$ of the total gravitational wave strain), there exists the possibility of extracting the memory effect by statistical analysis from the cumulative data sourced by a collection of merger events. For instance, $\sim 90$ mergers similar to GW150914 yield an expected memory signal-to-noise ratio $\left\langle S / N_{\text {tot }}\right\rangle=5$, whereas $\left\langle S / N_{\text {tot }}\right\rangle=3$ is achieved by only $\sim 35$ events [20]. In light of the recent LIGO detections it seems probable that mergers of relevant size are relatively commonplace in the Universe, making the expected frequency of future gravitational wave detections sufficient for measuring the memory in the coming years.

Having discussed the prospects of measuring the gravitational wave memory effect as well as the role of the gauge symmetries, let us finally contrast the situation with the $\mathrm{U}(1)$ memory. There is clearly some analogy between the two but also a considerable difference. In ED one has the overall $\mathrm{U}(1)$ gauge invariance and no need to define any new symmetries. In gravity the relevant transformations are a small carefully defined subset of general coordinate transformations and motivate a new symmetry transformation at null infinity, the BMS group [21].

\section{COLOR MEMORY}

In nature there are no massless free colored particles; asymptotic states have a mass and do not propagate to null infinity. However, there is one context where one comes close: the wave function of a large nucleus in an infinite momentum frame, probed with some large scale phenomenon like deep inelastic scattering at large $Q^{2}$ and "small $x$ ", $x=p^{+} / P^{+}=$longitudinal momentum fraction of a parton in a nucleus. In an infinite momentum frame the fast degrees of freedom are effectively frozen by time dilatation and can be represented by a time independent color current. This sources classical color fields which describe dynamics of soft and dense small $x$ degrees of freedom. Classical field description is justified by large occupation numbers in $p, q$ phase space. However, classical fields are only an intermediate stage; in physics non-Abelian gauge theory is quantum theory, and physics comes from an ensemble average over classical fields sourced by an ensemble of sources.

\section{A. Color fields}

Let us first summarize the relevant color ${ }^{1}$ fields [22]. We use the flat space light cone coordinates with the mostly plus metric $d s^{2}=-2 d x^{+} d x^{-}+d x^{i} d x^{i}, x^{ \pm}=(t \pm x) / \sqrt{2}$,

\footnotetext{
${ }^{1}$ Color conventions are $D_{\mu}=\partial_{\mu}-i g A_{\mu}, A_{\mu}=A_{\mu}^{a} T_{a},\left[T_{a}, T_{b}\right]=$ $i f_{a b c} T_{c}, \quad T_{b c}^{a}=-i f_{a b c}$ for adjoint representation, $F_{\mu \nu}=$ $i / g\left[D_{\mu}, D_{\nu}\right]=\partial_{\mu} A_{\nu}-\partial_{\nu} A_{\mu}-i g\left[A_{\mu}, A_{\nu}\right]$, under a unitary gauge transformation $U(x) A_{\mu} \rightarrow A_{\mu}^{\prime}=U A_{\mu} U^{\dagger}+i / g U \partial_{\mu} U^{\dagger}, F_{\mu \nu} \rightarrow$ $F_{\mu \nu}^{\prime}=U F_{\mu \nu} U^{\dagger}$.
} 
$i=2,3, x_{T}^{2}=x^{i} x^{i}$, with the nucleus moving in the positive $x$ direction. In the limit of large $x \sim r$ the two transverse coordinates $x^{i}$ are effectively the same as the $\mathrm{S}^{2}$ angular coordinates $\theta_{A}$ (scaled by $r$ ).

The equation to be solved is

$$
D_{\mu} F^{\mu \nu}=J^{\nu}=\delta^{\nu+} \rho\left(x^{-}, x^{i}\right) .
$$

Here $\rho$ is the color current of a nucleus moving in the $x$ direction in the infinite momentum frame. It is crucial for the following that there is no $x^{+}$dependence; there is no time dependence due to infinite time dilatation. In contrast to the $\mathrm{U}(1)$ case, the formulation is not gauge invariant, only gauge covariant. So we have to fix the gauge, and the usual choice is the light cone gauge $A^{-}=-A_{+}=0$. Then a current with only + component and no $x^{+}$ dependence automatically satisfies $D_{\mu} J^{\mu}=\partial_{+} J^{+}=0$, as required by (3.1).

However, $A^{-}=0$ is not yet complete gauge fixing, and one can fix further either $A^{i}=0$ [Eq. (3.3)] or $A^{+}=0$ [Eq. (3.8)]. The former is called the covariant gauge (COV), since in it automatically $\partial_{\mu} A^{\mu}=0$, and the latter, in unfortunate terminology, the light cone gauge (LC). In both of these gauges $F^{-+}=F^{-i}=0$ while only $F^{+i}$ is non-zero, $i=2,3$.

One can formally avoid gauge fixing $A^{-}$to zero by integrating $\mathrm{J}^{+}\left(x^{+}, x^{-}, x^{i}\right)$ from the matrix equation,

$$
\begin{aligned}
D_{+} J^{+} & =\partial_{+} J^{+}\left(x^{+}, x^{-}, x^{i}\right)-i g A^{-}\left(x^{+}, x^{i}\right) J^{+}\left(x^{+}, x^{-}, x^{i}\right) \\
& =0 .
\end{aligned}
$$

This is clearly exceedingly complicated and anyway useless since for physical applications one also has to include quantum fluctuations. This leads to an ensemble of color densities $\rho_{a}$, the distribution of which is determined by a renormalization group equation [9].

The covariant gauge (COV) corresponds to the gauge fixing,

$A^{\mu}=\left(A^{+}\left(x^{-}, x^{i}\right), 0,0,0\right), \quad A_{\mu}=\left(0,-A^{+}\left(x^{-}, x^{i}\right), 0,0\right)$,

for the vector potential. The absence of $x^{+}$dependence means that $\partial_{\mu} A^{\mu}=\partial_{+} A^{+}=0$, and the fact that $A^{\mu}$ has only one nonzero component implies that the cross term in $F_{\mu \nu}$ disappears. With the ansatz (3.3) the field tensor, in the $\left(x^{+}, x^{-}, x^{i}\right)$ basis, simply is

$F_{\mu \nu}=\left(\begin{array}{ccc}0 & 0 & 0 \\ 0 & 0 & \partial_{i} A^{+}\left(x^{-}, x^{i}\right) \\ 0 & -\partial_{i} A^{+}\left(x^{-}, x^{i}\right) & 0\end{array}\right)$

$(\mathrm{COV})$.
The only nonzero component of the field tensor thus is $F_{-i}$ while $F_{+i}=0$. The latter implies that $F_{t i}=-F_{x i}$ so that, writing $F_{t i}=E_{i}, F_{i j}=-\epsilon_{i j k} B^{k}$,

$$
E_{i}=\epsilon_{x i j} B_{j} \equiv \epsilon_{i j} B_{j}, \quad \epsilon_{23}=1,
$$

and

$F_{-i}=1 / \sqrt{2}\left(F_{t i}-F_{x i}\right)=\sqrt{2} E_{i}=\partial_{i} A^{+}\left(x^{-}, x^{i}\right)$.

Altogether we have $E_{i}=\epsilon_{i j} B^{j}, \epsilon_{23}=1, E_{i} B^{i}=0$, i.e., mutually orthogonal color electric and magnetic fields, a good analogy for electromagnetic radiation. The relation of the fields to the color current is obtained by solving $A^{+}$ from

$D_{\mu} F^{\mu+}=D_{i} F^{i+}=\partial_{i} \partial^{i} A^{+}=\partial_{i}^{2} A^{+}\left(x^{-}, x^{i}\right)=\rho\left(x^{-}, x^{i}\right)$,

i.e., by inverting the $2 \mathrm{D}$ transverse Poisson equation.

In the light cone gauge (LC) one asks for a potential of the form,

$$
A^{\mu}=\left(0,0, A^{i}\left(x^{-}, x^{j}\right)\right) .
$$

This is related to the previous by transforming $A^{+}$to zero in (3.3) by using the gauge transformation matrix,

$$
\partial_{-} U^{\dagger}\left(x^{-}, x^{i}\right)=-i g A^{+}\left(x^{-}, x^{i}\right) U^{\dagger}\left(x^{-}, x^{i}\right),
$$

which is solved by the path ordered exponential,

$U\left(x^{-}, x^{i}\right)=P \exp \left[i g \int_{0}^{x^{-}} d y^{-} A^{+}\left(y^{-}, x^{i}\right)\right] U\left(0, x^{i}\right)$.

Because there is no $x^{+}$dependence, $A^{-} \rightarrow U \partial_{+} U^{\dagger}=0$, no $A^{-}$is generated. For the transverse components one has

$$
A^{i}\left(x^{-}, x^{i}\right)=i / g U \partial_{i} U^{\dagger} .
$$

The transverse potential thus is gauge equivalent to vacuum; the transverse field tensor vanishes

$$
F_{i k}^{a}=\partial_{i} A_{k}^{a}-\partial_{k} A_{i}^{a}+g f_{a b c} A_{i}^{b} A_{k}^{c}=0 .
$$

Altogether the field tensor is

$$
F_{\mu \nu}=\left(\begin{array}{ccc}
0 & 0 & 0 \\
0 & 0 & \partial_{-} A^{i}\left(x^{-}, x^{i}\right) \\
0 & -\partial_{-} A^{i}\left(x^{-}, x^{i}\right) & 0
\end{array}\right)
$$


The electric field is

$$
\begin{aligned}
F_{-i} & =\sqrt{2} E_{i}^{\mathrm{LC}}=\partial_{-} A^{i}=i / g \partial_{-}\left(U \partial_{i} U^{\dagger}\right) \\
& =U \partial_{i} A^{+} U^{\dagger}=\sqrt{2} U E_{i}^{\mathrm{COV}} U^{\dagger},
\end{aligned}
$$

as should. So we have symmetrically, depending on gauge, either $\sqrt{2} E_{i}^{\mathrm{COV}}=\partial_{i} A^{+}\left(x^{-}, x^{i}\right)$ or $\sqrt{2} E_{i}^{\mathrm{LC}}=\partial_{-} A^{i}\left(x^{-}, x^{i}\right)$.

\section{B. Memory as a transverse kick}

We now have the classical color radiation fields-in a fixed $A^{-}=0$ gauge - and the next task is to formulate the analogue of the memory equation (2.13); i.e., how a transverse momentum kick of a test quark can be computed. For this we need a generalization of the Lorentz force and the equations of motion of a colored test particle in a known color field, the Wong equations [23-25]. The same equations have recently been used [11] in a study of collisions of a nucleus with a static test quark; the emphasis there was on the gluon radiation caused by the acceleration of the quark.

Generalizing the electrodynamic action for a point particle following the path $x^{\mu}=x^{\mu}(\tau)$ by introducing a color vector $Q^{a}(\tau)$, the Wong equations can be derived from the action,

$$
\begin{aligned}
S= & \int d \tau\left[-m \frac{d s}{d \tau}+g \dot{x}^{\mu}(\tau) A_{\mu}^{a}\left(x^{\alpha}(\tau)\right) Q_{a}(\tau)\right] \\
= & \int d \tau L\left[x^{\mu}(\tau), \dot{x}^{\mu}(\tau)\right] \\
= & -m \int d s+\int d^{4} x\left[\int d \tau \delta^{4}\left(x^{\mu}-x^{\mu}(\tau)\right) g Q_{a}(x) u^{\mu}\right] \\
& \times A_{\mu}^{a}(x) .
\end{aligned}
$$

For a given vector potential $A_{\mu}^{a}$ the equations are extremal equations for a particle path $x^{\mu}=x^{\mu}(\tau)$. Defining first,

$$
p^{\mu}=m u^{\mu}=m \frac{d x^{\mu}}{d \tau},
$$

they are $\left(Q \cdot F \equiv Q_{a} F^{a}=2 \operatorname{Tr} Q F\right)$,

$\frac{d p^{\mu}}{d \tau}=g Q \cdot F^{\mu \nu} \frac{d x_{\nu}}{d \tau}, \quad \frac{d Q^{a}}{d \tau}=-g f_{a b c} u^{\mu} A_{\mu}^{b} Q^{c}$.

Here the proper time dependence of $Q(\tau)$ follows elegantly from demanding that the extremal equations derived from the Lagrangian in (3.15) give the correct nonAbelian cross term in $F^{\mu \nu}$ in the first equation. Note that the equation for $p_{\mu}$ explicitly conserves the mass shell condition $p_{\mu} p^{\mu}=-m^{2}$.

The equation for $\dot{Q} \equiv d Q / d \tau$ also follows from the conservation law $D_{\mu} J^{\mu}=0$ for the current,

$$
J^{\mu}=\int d \tau Q(\tau) u^{\mu}(\tau) \delta^{4}(x-x(\tau))
$$

In a matrix form $\left(u^{\mu} \partial_{\mu}=\partial_{\tau}\right)$,

$\dot{Q}-i g u^{\mu} A_{\mu} Q=u^{\mu}\left(\partial_{\mu}-i g A_{\mu}\right) Q=u^{\mu} D_{\mu} Q=0$.

This first order matrix equation can be integrated to give

$$
Q(\tau)=P \exp \left[i g \int_{0}^{\tau} d x^{\mu} A_{\mu}(x)\right] Q(0) .
$$

Consider then the Lorentz force equation for $\mu=-, i,+$. For $\mu=-$ one simply has $F^{-+}=F^{-i}=0$ and

$\frac{d p^{-}}{d \tau}=0 \Rightarrow \frac{p^{-}}{m}=u^{-}=\frac{d x^{-}}{d \tau}=$ constant $\Rightarrow x^{-}(\tau)=u^{-} \tau$.

The fact that the $\mu=-$ component is so simple basically follows from the time or $x^{+}$independence of the gluon radiation burst in (3.1). The Lorentz force equation trivially conserves $p^{2}=-2 p^{+} p^{-}+p_{i}^{2}=-m^{2}$ from which

$$
p^{-} \frac{d p^{+}}{d \tau}=p_{i} \frac{d p^{i}}{d \tau} .
$$

Thus only the equation for $p^{i}$ is needed. In the $A^{-}=0$ gauge,

$$
\begin{aligned}
\frac{d p^{i}(\tau)}{d \tau}=m \frac{d^{2} x^{i}(\tau)}{d \tau^{2}} & =g Q \cdot F^{i+} \frac{d x_{+}}{d \tau} \\
& =-g u^{-} Q(\tau) \cdot \partial_{-} A^{i}\left(x^{-}, x^{k}(\tau)\right) \\
& =-g u^{-} \sqrt{2} Q(\tau) \cdot E^{i}\left(x^{-}, x^{k}(\tau)\right),
\end{aligned}
$$

where we inserted $x_{+}=-x^{-}$and remember that $x^{-}=u^{-} \tau$. Eq. (3.23) with the color electric field is obviously the analogue of the simple equation $\ddot{x}=e E_{x}$ in the Abelian ED case (2.13).

Together with Eq. (3.17) for $Q(\tau)$, Eq. (3.23) is a very complicated second order differential equation for the transverse coordinate $x^{i}(\tau), i=2,3$. First, we are given a color distribution $\rho_{a}\left(x^{-}, x^{i}\right)$ in the infinite momentum wave function of a nucleus; one may imagine a Gaussian in all variables. It serves as the inhomogeneous source term of a 2D Poisson equation (3.7) for the potential $A^{+}\left(x^{-}, x^{i}\right)$. Using this one can define the path ordered exponential $U\left(x^{-}, x^{i}\right)$ in Eq. (3.10) which, via Eq. (3.11) gives the transverse vector potential $A^{i}\left(x^{-}, x^{i}\right)$. When this background field is given one can solve the rotation of $Q(\tau)$ from (3.20). All these equations depend implicitly on the quantity to be solved, $x^{i}(\tau)$. Assuming the test quark is 
initially at rest, $x^{i}(0)=x_{0}^{i}, x^{i \prime}(0)=0$, one can, in principle, solve $x^{i}(\tau)$ and $p^{i}(\tau)$. Yang-Mills memory then is simply given by the total transverse kick,

$$
\Delta p^{i}=p^{i}\left(\tau_{f}\right)-p^{i}(0)
$$

The above computation was carried out in the $A^{-}=0$ gauge. Transformation within the two gauges in this class is

$$
\begin{aligned}
F_{\mathrm{COV}}^{i+} & =U^{\dagger} F_{\mathrm{LC}}^{i+} U=\partial_{i} A_{\mathrm{COV}}^{+}=U^{\dagger} \partial_{-} A_{i}^{\mathrm{LC}} U, \\
Q_{\mathrm{LC}} & =U Q_{\mathrm{COV}} U^{\dagger},
\end{aligned}
$$

and the result, which is $\sim \operatorname{Tr} Q F^{i+}$, is explicitly invariant and physical under these transformations. Restoring $A^{-}$as in (3.2) is also possible, but does not change the fact that memory is defined in a fixed gauge. In ED, in the formulation of [2], the gauge transformations $U$ simply disappear from the definition.

\section{SIMPLIFICATION IN QUANTUM THEORY}

To integrate (3.23) and to use the remaining piece of information, the $\tau$ or $x^{-}$derivative of $Q$ in (3.17), we manipulate (3.23) as follows:

$$
\begin{aligned}
\frac{d p^{i}\left(x^{-}\right)}{d x^{-}} & =-g\left[\partial_{-}\left(Q_{a}\left(x^{-}\right) A_{a}^{i}\right)-A_{a}^{i} \partial_{-} Q_{a}\right] \\
& =-g\left[\frac{d\left(Q_{a}\left(x^{-}\right) A_{a}^{i}\right)}{d x^{-}}+g f_{a b c} A_{i}^{a} A_{k}^{b} Q_{c}\left(x^{-}\right) \frac{d x^{k}}{d x^{-}}\right] .
\end{aligned}
$$

In the present gauge $A_{ \pm}=0$ and in the sum in (3.17) only the spatial term $u^{k} A_{k}^{b}$ remains. In (4.1) we could replace $g f_{a b c} A_{i}^{a} A_{k}^{b}=-\partial_{i} A_{k}^{c}+\partial_{k} A_{i}^{c}$ (since $F_{i k}=0$ ), but this complicated term does not vanish.

In the quantum theory of color glass condensate (CGC) the situation is actually much simpler. There one does not compute the fields for a fixed color distribution $\rho_{a}$ in (3.1), but integrates over a distribution thereof in order to compute expectation values; see, e.g., [9] Sec. II. These are diagonal in color. On the average, color density vanishes, $\left\langle\rho_{a}\right\rangle=0$, and the starting point is the charge density correlator,

$$
\left\langle\rho_{a}\left(x^{-}, x^{i}\right) \rho_{b}\left(y^{-}, y^{j}\right)\right\rangle=\delta_{a b} \delta\left(x^{-}-y^{-}\right) \delta^{(2)}(x-y) \lambda_{A}\left(x^{-}\right),
$$

where $\lambda_{A}\left(x^{-}\right)$is the average color charge squared of valence quarks per color and per volume (so that its integral over $x^{-}$is the average transverse density). From this one can compute $\left\langle A_{a}^{+}\left(x^{-}, x^{i}\right) A_{b}^{+}\left(y^{-}, y^{j}\right)\right\rangle$ and further correlators of the type $\left\langle A_{i}^{a} A_{j}^{b}\right\rangle$. These are all diagonal, $\sim \delta_{a b}$. When applied to (4.1), the last term in it vanishes, due to the antisymmetry of $f_{a b c}$. Thus we can immediately integrate and find

$$
p^{i}\left(x^{-}\right)=m \frac{d x^{i}}{d \tau}=-g Q_{a}\left(x^{-}\right) A_{a}^{i}\left(x^{-}, x^{i}\left(x^{-}\right)\right) .
$$

We already have solved $x^{-}(\tau)=u^{-} \tau$, and the transverse coordinate $x^{i}=x^{i}(\tau)$ can also be solved from here. Finally, the mass shell condition (3.22) gives, after integration over $\tau$,

$$
p^{+}(\tau)=m \frac{d x^{+}}{d \tau}=\frac{\left(g Q \cdot A^{i}\right)^{2}}{2 p_{-}}+\mathrm{const}=\frac{p_{i}^{2}(\tau)+m^{2}}{2 p^{-}} .
$$

The above is a great simplification relative to (4.1), but in full quantum theory the coordinates disappear and what matters is the expectation value [9],

$$
p_{T}^{2}=\left\langle p^{i} p^{i}\right\rangle=g^{2} Q_{b} Q_{b}\left\langle A_{a}^{i} A_{a}^{i}\right\rangle .
$$

The signal of color memory is thus $p_{T} \sim g Q\left|A^{i}\right|$, the magnitude of the transverse color field in the gauge $A^{-}=0$, generated by the passing of the nucleus. This generalizes the memory in ED, $\Delta p_{B}=q\left(A_{B}\left(u_{f}\right)-A_{B}\left(u_{i}\right)\right)$ in the gauge $A_{u}=0$, derived in Eqs. (2.13) and (2.15). Deriving the physical magnitude of the kick is one of the achievements of the theory of CGC, especially for very large nuclei. A dense system of gluons saturates and generates a dynamical large scale, the saturation scale $Q_{s} \sim$ few $\mathrm{GeV}$. The physical magnitude of the kick then is, for dimensional reasons, $\sim Q_{s}$.

Since (4.5) is the main result of this article, it might be useful to add some detail on how its magnitude is computed, in the simplest possible way. One assumes that the nucleus is infinitely Lorentz contracted, $x^{-}$dependence is $\delta\left(x^{-}\right)$, and that the color density of the nucleus fluctuates according to the Gaussian distribution,

$$
W\left[\rho_{a}(\mathbf{x})\right]=\exp \left[-\int d^{2} z \frac{1}{2 \lambda}\left(\rho_{a}(\mathbf{z})\right)^{2}\right],
$$

where only the dependence on the transverse coordinate $\mathbf{z}$ is needed. Average charge then vanishes and quadratic correlators are given by (4.2) with $x^{-}$dependence removed (only $x^{-}=0$ contributes). Physics, properties of the nuclear wave function, is embedded in the constant $\lambda$. According to (3.11) $A_{i}(\mathbf{x})$ is simply related to the Wilson line (3.10) and the expectation value of the correlator becomes

$$
\begin{aligned}
\left\langle\operatorname{Tr} A_{i}(\mathbf{x}) A_{j}(\mathbf{y})\right\rangle_{\rho} & =\frac{1}{2}\left\langle A_{i}^{a} A_{j}^{a}\right\rangle_{\rho} \\
& =\frac{1}{g^{2}}\left\langle\operatorname{Tr}\left(U \partial_{i} U^{\dagger}(\mathbf{x}) \partial_{j} U U^{\dagger}(\mathbf{y})\right\rangle_{\rho} .\right.
\end{aligned}
$$


We need the magnitude of $A_{i}$ at a point, so $\mathbf{x} \rightarrow \mathbf{y}$, $U^{\dagger} U=1$, and one has to compute in this limit,

$$
\left\langle\operatorname{Tr} A_{i}(\mathbf{x}) A_{j}(\mathbf{y})\right\rangle_{\rho}=\frac{1}{g^{2}} \partial_{i}^{x} \partial_{j}^{y}\left\langle\operatorname{Tr}\left(U^{\dagger}(\mathbf{x}) U(\mathbf{y})\right\rangle_{\rho}\right.
$$

$U$ is $\sim \exp \left(-i g A^{+}\right)$by $(3.10)$ and $A^{+}=\left(1 / \partial_{i}^{2}\right) \rho$ by the Poisson equation (3.7) so that the product $U U^{\dagger}$ is exponential of a linear functional of $\rho$ and the Gaussian integral over the weight function (4.6) can be carried out. The 2D Poisson equation has a logarithmic divergence, though, and cutting this away by $1 / k_{T}^{2} \rightarrow 1 /\left(k_{T}^{2}+m^{2}\right)$, the result is

$$
\begin{aligned}
\left\langle\operatorname{Tr}\left(U^{\dagger}(\mathbf{x}) U(\mathbf{y})\right\rangle_{\rho}\right. & =\exp \left[-\frac{\lambda g^{2} N_{c}}{4 \pi m^{2}}\left(1-m r_{T} K_{1}\left(m r_{T}\right)\right)\right] \\
& \approx \exp \left[-Q_{s}^{2} \frac{r_{T}^{2}}{4 \pi} \log \frac{1}{r_{T} \Lambda}\right]
\end{aligned}
$$

where $Q_{s}^{2}=\frac{1}{2} \lambda g^{2} N_{c}, r_{T}=|\mathbf{x}-\mathbf{y}|, \Lambda=\frac{1}{2} e^{\gamma_{E}-\frac{1}{2} m}$ and we have taken the limit $m \rightarrow 0$. The final step of taking the derivatives in (4.8) leads to

$$
\lim _{\mathbf{x} \rightarrow \mathbf{y}}\left\langle A_{i}^{a}(\mathbf{x}) A_{i}^{a}(\mathbf{y})\right\rangle=Q_{s}^{2} \frac{1}{g^{2} \pi} \lim _{r_{T} \rightarrow 0} \log \frac{1}{r_{T} \Lambda},
$$

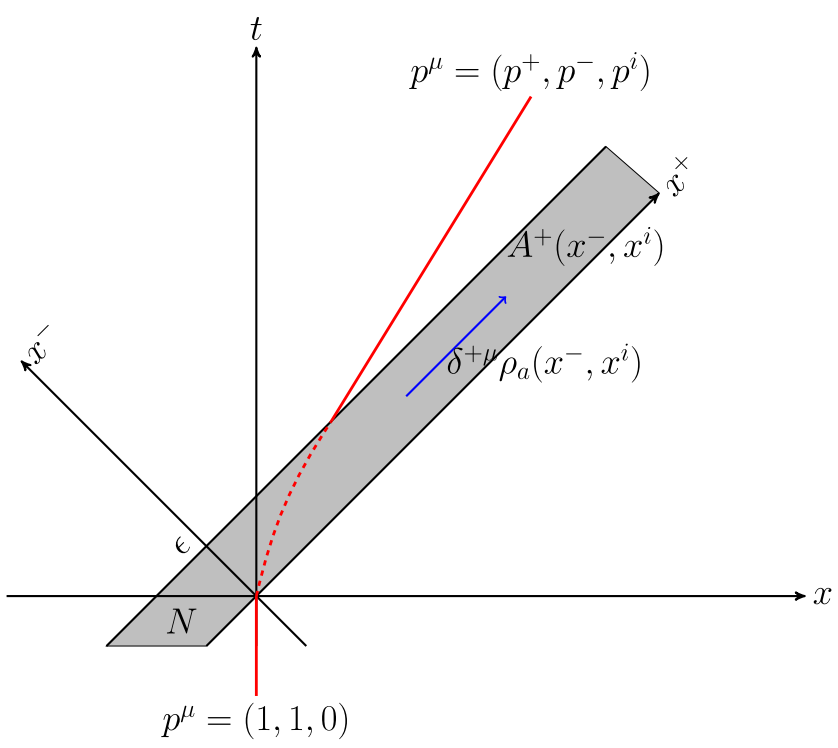

FIG. 2. Interaction of a nuclear Yang-Mills field and a test quark. The nucleus $N$ is represented by a color current $J^{+}=$ $\rho\left(x^{-}, x^{i}\right)$ and associated classical YM field $A^{+}$. There is no dependence on the LC time $x^{+}$. The field extends over the range $0<x^{-}<\epsilon$ and $\epsilon \rightarrow 0$ with increasing energy. In the transverse gauge $A^{\mu}=\left(0,0, A^{i}=i / g U \partial_{i} U^{\dagger} \theta\left(x^{-}\right)\right)$, fields in $0<x^{-}<\epsilon$ are given in [22]. The collision with the test quark at rest accelerates the quark to transverse momentum $p^{i}$; this is the YM memory. Transverse coordinates are not shown in the figure. a result proportional to $Q_{s}^{2}$ but now divergent at small distances. This reflects the small size of the probe, a single quark, the kick of which one is studying. In momentum space this would correspond to the tail at large momentum. Dynamics at small distances or at large momenta has to cut off the divergence, but the above outline of a computation is offered here as an illustration of the difficulties testing Yang-Mills memory in a nuclear environment has to face.

It is interesting to ask if the color memory effect could be experimentally verified. The observable linked most naturally with the kick (4.5) is the color dipole cross section [10], and the prospects for its extraction in the future Electron-Ion Collider is discussed in [26]. The detection seems a plausible scenario, albeit in a highly convoluted environment.

Finally, let us point out that in principle, a color rotation of the quark could also be a signal of the memory [6]. However, in [11] it was shown that in the relevant limit of very high energies, $\epsilon \rightarrow 0$ in Fig. 2, this rotation vanishes.

\section{CONCLUSIONS}

We have discussed the memory effect in Abelian and non-Abelian gauge theories. In Abelian electrodynamics the discussion can be entirely formulated using gauge invariant quantities, electric and magnetic fields [3]. The effect manifests itself as a change in the transverse momentum of a test charge caused by the passing of the radiation pulse. It has two components, an ordinary kick caused by the change in the radial component of the electric field and a null kick caused by flux of charge to null infinity. The latter, more interesting, part requires that there be massless charged particles since massive ones do not get to null infinity. Such ones do not exist but could exist so this is a useful conceptual exercise as an analogy to gravity; there certainly massless particles carry energy to null infinity.

The U(1) memory could be analyzed entirely in terms of gauge invariant variables. However, one can also fix the gauge at null infinity, and one thus has a means of determining physically a magnitude of a gauge transformation [4]. This is a "large" (nonzero) gauge transformation at asymptotic infinity. One can formulate this as a symmetry of ED at infinity, but it is essentially just $\mathrm{U}(1)$ gauge invariance.

In the non-Abelian case there in nature are no classical fields of the type used in the discussion of $\mathrm{U}(1)$ memory, QCD is in the confinement phase; gluons dress themselves to glueballs and do not propagate to null infinity. However, classical YM fields supplemented with their quantum fluctuations are ubiquitous in discussions of dynamics of large nuclei; in certain experimental conditions they become very dense systems with large occupation numbers so that use of classical fields is motivated. Large transverse densities produce effectively a large energy scale, 
saturation scale, so that the coupling becomes-optimistically - weak. In this framework, the theory of color glass condensate, one can immediately find a generalization of the key U(1) memory equation (2.15), in which a physical quantity, transverse kick, is expressed as color times gauge potential in a certain gauge. Initially one has a linear equation of the type in (4.3), but its expectation value vanishes, and what matters is the quantum expectation value of its square in (4.5).

The discussion of memory in YM theory is thus not manifestly gauge invariant, and physical results are obtained only after gauge fixing in quantum theory. It is thus not useful as an analogue model of gravitational radiation. Rather the other way round, it can be used to reinterpret some well-known properties of the theory of CGC.

\section{ACKNOWLEDGMENTS}

Miika Sarkkinen is supported by the Finnish Cultural Foundation. We thank L. McLerran, T. Lappi, and R. Paatelainen for discussions on color glass condensate and L. Bieri, D. Garfinkle, D. Nichols, and B. Oblak for discussions on gravitational radiation memory, in particular at a Solvay workshop in May 2018.
[1] C. M. Will and A. G. Wiseman, Gravitational radiation from compact binary systems: Gravitational wave forms and energy loss to second postNewtonian order, Phys. Rev. D 54, 4813 (1996).

[2] L. Bieri and D. Garfinkle, Perturbative and gauge invariant treatment of gravitational wave memory, Phys. Rev. D 89, 084039 (2014).

[3] L. Bieri and D. Garfinkle, An electromagnetic analogue of gravitational wave memory, Classical Quantum Gravity 30, 195009 (2013).

[4] J. Winicour, Global aspects of radiation memory, Classical Quantum Gravity 31, 205003 (2014).

[5] L. Bieri and D. Garfinkle, Neutrino radiation showing a christodoulou memory effect in general relativity, Ann. Henri Poincare 16, 801 (2015).

[6] M. Pate, A. M. Raclariu, and A. Strominger, Color Memory: A Yang-Mills Analog of Gravitational Wave Memory, Phys. Rev. Lett. 119, 261602 (2017).

[7] D. Kapec, M. Pate, and A. Strominger, New symmetries of QED, Adv. Theor. Math. Phys. 21, 1769 (2017).

[8] G. 't Hooft, Topological aspects of quantum chromodynamics, arXiv:hep-th/9812204.

[9] E. Iancu, A. Leonidov, and L. McLerran, The color glass condensate: An introduction, arXiv:hep-ph/0202270.

[10] A. Ball, M. Pate, A. M. Raclariu, A. Strominger, and R. Venugopalan, Measuring color memory in a color glass condensate at electron-ion colliders, Ann. Phys. (Amsterdam) 407, 15 (2019).

[11] K. Kajantie, L. D. McLerran, and R. Paatelainen, Gluon radiation from a classical point particle, arXiv:1903.01381.

[12] D. Bodeker, G. D. Moore, and K. Rummukainen, ChernSimons number diffusion and hard thermal loops on the lattice, Phys. Rev. D 61, 056003 (2000).

[13] M. D’Onofrio, K. Rummukainen, and A. Tranberg, Sphaleron Rate in the Minimal Standard Model, Phys. Rev. Lett. 113, 141602 (2014).

[14] A. Kehagias and A. Riotto, BMS in Cosmology, J. Cosmol. Astropart. Phys. 05 (2016) 059.
[15] Y.Z. Chu, More On cosmological gravitational waves and their memories, Classical Quantum Gravity 34, 194001 (2017).

[16] A. Kurkela, Initial state of heavy-ion collisions: Isotropization and thermalization, Nucl. Phys. A956, 136 (2016).

[17] K. Boguslavski, A. Kurkela, T. Lappi, and J. Peuron, Spectral function for overoccupied gluodynamics from real-time lattice simulations, Phys. Rev. D 98, 014006 (2018).

[18] L. N. Mihaila, J. Salomon, and M. Steinhauser, Gauge Coupling Beta Functions in the Standard Model to Three Loops, Phys. Rev. Lett. 108, 151602 (2012).

[19] A. Strominger, Lectures on the infrared structure of gravity and gauge theory, arXiv:1703.05448.

[20] P. D. Lasky, E. Thrane, Y. Levin, J. Blackman, and Y. Chen, Detecting Gravitational-Wave Memory with LIGO: Implications of GW150914, Phys. Rev. Lett. 117, 061102 (2016).

[21] R. Sachs, Asymptotic Symmetries in Gravitational Theory, Phys. Rev. 128, 2851 (1962).

[22] J. P. Blaizot and Y. Mehtar-Tani, The classical field created in early stages of high energy nucleus-nucleus collisions, Nucl. Phys. A818, 97 (2009).

[23] S. K. Wong, Field and particle equations for the classical Yang-Mills field and particles with isotopic spin, Nuovo Cimento A 65, 689 (1970).

[24] P. F. Kelly, Q. Liu, C. Lucchesi, and C. Manuel, Classical transport theory and hard thermal loops in the quark-gluon plasma, Phys. Rev. D 50, 4209 (1994).

[25] J. Jalilian-Marian, S. Jeon, and R. Venugopalan, Wong's equations and the small $\mathrm{x}$ effective action in QCD, Phys. Rev. D 63, 036004 (2001).

[26] E. C. Aschenauer S. Fazio, J. H. Lee, H. Mäntysaari, B. S. Page, B. Schenke, T. Ullrich, R. Venugopalan, and P. Zurita, The electronion collider: Assessing the energy dependence of key measurements, Rep. Prog. Phys. 82, 024301 (2019). 